\title{
A qualitative study of contemporary secure mental health services: women service users' views in England.
}

Dr Tammi Walker ${ }^{1,2}$ Professor Jenny Shaw, ${ }^{3}$ Dr Dawn Edge, ${ }^{2}$ Dr Jane Senior, ${ }^{4}$ Professor Matthew Sutton, ${ }^{5}$ Dr Rachel Meacock, ${ }^{5}$ Dr Hannah Wilson,,${ }^{2} 6$ Dr Louisa McNair, 2,7 Heather Mitchell, ${ }^{29}$ Dr Kerry Gutridge, ${ }^{2}$ and Professor Kathryn M. Abel. ${ }^{2,9}$

\section{Dr Tammi Walker, PhD (corresponding author)}

${ }^{1}$ Department of Psychology, School of Human \& Health Sciences, University of Huddersfield, Edith Key Building, Queensgate, Huddersfield, HD1 3DH, United Kingdom. t.walker@hud.ac.uk

ORCiD id: 0000-0001-7446-8771

${ }^{2}$ Centre for Women's Mental Health, School of Health Sciences, Faculty of Biology, Medicine and Health, University of Manchester, Manchester Academic Health Science Centre, Jean McFarlane Building, Manchester, M13 9PL, United Kingdom.

\section{Professor Jenny Shaw}

${ }^{3}$ Centre for Mental Health and Safety, School of Health Sciences, Faculty of Biology, Medicine and Health, University of Manchester, Manchester Academic Health Science Centre, Jean McFarlane Building, Manchester, M13 9PL. jennifer.j.shaw@manchester.ac.uk

ORCiD id: 0000-0003-2569-7687 


\section{Dr Dawn Edge}

${ }^{2}$ Centre for Women's Mental Health, School of Health Sciences, Faculty of Biology, Medicine and Health, University of Manchester, Manchester Academic Health Science Centre, Jean McFarlane Building, Manchester, M13 9PL, United Kingdom. dawn.edge@manchester.ac.uk

ORCiD id: 0000-0003-1139-6613

\section{Dr Jane Senior, PhD}

${ }^{3}$ Centre for Mental Health and Safety, School of Health Sciences, Faculty of Biology, Medicine and Health, University of Manchester, Manchester Academic Health Science Centre, Jean McFarlane Building, Manchester, M13 9PL, United Kingdom. jane.senior@manchester.ac.uk

ORCiD id: 0000-0002-7133-4898

\section{Professor Matthew Sutton, PhD}

${ }^{4}$ Manchester Centre for Health Economics, Institute of Population Health, University of Manchester, Manchester Academic Health Science Centre, Jean McFarlane Building, Manchester, M13 9PL, United Kingdom. matt.sutton@manchester.ac.uk

ORCiD id: 0000-0002-6635-2127

\section{Dr Rachel Meacock, PhD}


${ }^{4}$ Manchester Centre for Health Economics, Institute of Population Health, University of Manchester, Manchester Academic Health Science Centre, Jean McFarlane Building, Manchester, M13 9PL, United Kingdom. rachel.meacock@ manchester.ac.uk

ORCiD id: 0000-0001-8933-5058

\section{Dr Hannah Wilson, DClinPsy}

${ }^{2}$ Centre for Women's Mental Health, School of Health Sciences, Faculty of Biology, Medicine and Health, University of Manchester, Manchester Academic Health Science Centre, Jean McFarlane Building, Manchester, M13 9PL, United Kingdom.

${ }^{5}$ Lancashire Care NHS Foundation Trust, Preston, PR5 6AW, United Kingdom. Hannah.wilson@lancashirecare.nhs.uk

\section{Dr Louisa McNair, DClinPsy}

${ }^{2}$ Centre for Women's Mental Health, School of Health Sciences, Faculty of Biology, Medicine and Health, University of Manchester, Manchester Academic Health Science Centre, Jean McFarlane Building, Manchester, M13 9PL, United Kingdom.

${ }^{6}$ Cheshire and Wirral Partnership NHS Foundation Trust, Chester, CH2 1BQ, United Kingdom.

Louisa.mcnair@cwp.nhs.uk

\section{Dr Kerry Gutridge, PhD}

${ }^{2}$ Centre for Women's Mental Health, School of Health Sciences, Faculty of Biology, Medicine and Health, University of Manchester, Manchester Academic Health Science Centre, Jean McFarlane Building, Manchester, M13 9PL, United Kingdom. kerry.gutridge@manchester.ac.uk 
ORCiD id: 0000-0001-9705-9102

\section{Ms Heather Mitchell, BA}

${ }^{2}$ Centre for Women's Mental Health, Faculty of Biology, Medicine and Health, University of Manchester, Manchester Academic Health Science Centre, Jean McFarlane Building, Manchester, M13 9PL, United Kingdom

${ }^{7}$ Greater Manchester Mental Health NHS Foundation Trust, Prestwich, M25 3BL, United Kingdom.

heather.mitchell-2@ postgrad.manchester.ac.uk

ORCiD id: 0000-0002-5244-6528

\section{Professor Kathryn M. Abel, PhD MA MB ChB}

${ }^{2}$ Centre for Women's Mental Health, Faculty of Biology, Medicine and Health, University of Manchester, Manchester Academic Health Science Centre, Jean McFarlane Building, Manchester, M13 9PL, United Kingdom

${ }^{7}$ Greater Manchester Mental Health NHS Foundation Trust, Prestwich, M25 3BL, United Kingdom. Kathryn.M.Abel@manchester.ac.uk

ORCiD id: 0000-0003-3538-8896

The research was conducted at the University of Manchester.

\section{Competing interests}

The authors declare that they have no competing interests. 


\section{Funding}

This work was supported by the Department of Health: 'A clinical, economic and operational evaluation of the pilot Women's Enhanced Medium Secure Services (WEMSS), funded by the Department of Health's National Oversight Group (NOG). The views expressed in this paper represent those of the authors and do not necessarily represent those of the funders. 


\title{
A qualitative study of contemporary secure mental health services: women service users' views in England.
}

\begin{abstract}
Women's Enhanced Medium Secure Services (WEMSS) was a concept borne out of findings that an inappropriate number of women were being held in high secure services, despite not fulfilling high secure criterion. A qualitative study of women service users' views of living in WEMSS and comparator medium secure services (MSS) in the UK is presented. Sixteen service users participated in semi-structured interviews. Thematic analysis identified four main themes: experiences of current placement versus previous placements, relationships with staff, challenges of living with other women and having a voice - being involved in care and treatment. The accounts reported suggest that women in WEMSS and standard MSS have very similar experiences of their service, relationships with staff, living with other women and involvement in care. This qualitative study suggests that women in WEMSS and comparable women in MSS have very similar perspectives on what works well in their current services and what is important to them. The main differences between WEMSS and MSS women's accounts were in relation to the amount of pre-transfer information they received and levels of staff support. Implications for practice are discussed, including service-user empowerment addressing barriers to relational security.
\end{abstract}

Key words: secure mental health; women; prison; qualitative 


\section{Introduction}

In the past decade, there have been significant changes in the provision of secure mental health care for women. Many of these changes occurred in response to a growing consensus that women's needs were not being met by secure mental health services in England (Reed, 1992). In 1994 (Howlett, 1994) and again in 2001 (Shaw, Davies \& Morey, 2001) it was reported that approximately $80 \%$ of women were being cared for in services with an unsuitable level of security. The Department of Health strategies (Department of Health, 2002; 2003; 2004) suggested that an inappropriate number of women were being held in high secure services when they did not fulfill the primary criterion of posing a 'grave and immediate danger'. In addition to calling for reduced high secure services, the pressing need to adequately address the specific needs of women in secure care was also raised (Department of Health, 2000).

By 2007, Ashworth and Broadmoor's high secure beds for women had closed and it was agreed that Rampton would serve as the only national high secure service for women. This gave rise to the concept and implementation of three pilot Women's Enhanced Medium Secure Services (WEMSS) which were developed to manage the transition of women out of high security and to provide an alternative model of care for women with complex mental health needs (Edge, 2005). The WEMSS model focused on improving services across four key areas: therapeutic engagement, quality of life, faster movement through the treatment pathway and increased relational security (Edge, Walker, Meacock \& Wilson, 2017). The focus on increased relational security rather than physical security was seen as central to the WEMSS model and to providing an effective therapeutic 
service for women who tend to have disturbed patterns of attachments and interpersonal relationships. Good relational security is achieved through detailed knowledge and understanding of service users, their histories and the reason for their current placement in secure services and is fostered by good service user-staff relationships and wider treatment programmes (Royal College of Psychiatrists, 2000).

Within the WEMSS model, focus was also placed on women's ability to exercise choice and make decisions about every aspect of their lives and the positive impact that this could have on their quality of life. Many women in secure care have a history of trauma, including sexual and physical abuse (Sahota et al., 2010; Walker \& Towl, 2016), which may have led to a sense of disempowerment. However, until the Department of Health published the guidelines ‘Mainstreaming Gender and Women's Mental Health: Implementation Guidance' (Department of Health, 2003), no recommendations had focused on the importance of empowering women and giving them a voice. WEMSS, therefore, aimed to deliver an explicitly gender-specific philosophy of care that was needs-led and focused on maximising women's potential for recovery (Edge, 2005).

Prior to the implementation of WEMSS, the Department of Health proposed that independent research was required to determine their success or failure (and therefore whether or not to roll them out), and should be conducted within five years. In particular, it was determined that WEMSS should be evaluated against Medium Secure Services (MSS) in both NHS and Independent Sectors. Although the small number of women in the three WEMSS pilots presented a methodological challenge, it was argued that the 
evaluation should involve collection of good quality quantitative and qualitative data which would be used to undertake a robust, holistic evaluation of the units in terms of clinical and cost-effectiveness (Edge, 2005).

Present Study

The current study focuses on the qualitative outputs of a larger evaluation study that used a series of case control studies involving women and staff in WEMSS and nonWEMSS facilities to compare: clinical, functional and social outcomes (the results of which are reported elsewhere; Edge et al., 2016). A semi-structured interview schedule was developed which aimed to explore and develop an understanding of what WEMSS women and non-WEMSS women in comparable standard MSS thought about their care and what they felt worked or did not work. This is the first study to report on service user experiences of WEMSS and MSS.

\section{Methods}

\section{Sample}

A purposive sample (Mays \& Pope, 1995) was selected for qualitative data collection. Purposive sampling is a form of non-probability sampling undertaken when strict levels of statistical reliability and validity are not required due to the exploratory nature of the research (Kidder, 1981). For the interviews with the women service users, a familiar staff member provided a brief description of the study, after a discussion with the Responsible Clinician. If participants volunteered, a Research Assistant met with them to answer any 
questions. Consent was obtained in accordance with research governance guidelines. Nine WEMSS women and seven non-WEMSS women service users were interviewed.

\section{Inclusion/exclusion criteria}

In order to take part in this study, participants needed to be over the age of 18 , able to provide written informed consent and not pose a risk to the researchers conducting interviews. Participants were excluded from the study if they did not meet any of the inclusion criteria (under 18 years of age, not able to provide written informed consent or posed a risk to researchers). This included a current mental state that did not allow reliable informed consent to be gained. The research team had intended to interview an additional one WEMSS woman and three non-WEMSS women but lack of informed consent, current risk or inappropriate mental state excluded these women.

The authors are keen not to disclose the settings involved because the relatively small number and restricted area over which the study occurred might enable identification of participants. The fieldwork took place over a period of six months (December 2010 to May 2011) at the three WEMSS pilot sites and non-WEMSS NHS medium secure sites.

\section{Procedure}

For the interviews, all participants were provided with an information sheet that contained an assurance of anonymity, information regarding the study, the possibility to withdraw and the voluntariness of participation. If they were willing to be interviewed, they signed the consent form and the interview took place. Consent was obtained in accordance with 
research governance guidelines, including consent for publication. The interviews were held at a date and time convenient to the participants and they all occurred at the hospital sites. Interviews were audio recorded and lasted between 30 and 45 minutes. Some service user interviews were conducted with a member of staff present, either at the request of staff or the service user.

\section{Interview structure}

A review of the literature was undertaken to identify key issues and to develop a semistructured interview schedule (Patton, 1990), which addressed the main research questions of the evaluation. Question development was informed by previous schedules developed by Parry-Crooke and Stafford (2009). The order of questions was based on recommendations from Miller \& Crabtree (1992) who suggest initial 'rapport-building' questions should be followed by more searching questions which require more detailed responses. This approach is particularly important when interviewing service users, advocates and carers (Edge, 2005; Walker \& Doyle, 2009). Before the full interviews were conducted, the schedule was piloted with one staff member and one service user, both from a WEMSS service. Amendments were made to the interviews based on comments from the ethics committee and feedback from the pilots.

The interview schedule consisted of questions about how they came to be in that particular service (such as 'How were you referred to this unit?'), what they thought of the services, (such as 'What do you think of the range of care/treatment which you can access?') their relationships with staff and other women in the services (such as 'How do you feel about 
your current relationships with staff?'), whether they felt in control of their lives (such as 'Do you feel in control of your own life?'), and any suggested changes or improvements (such as 'Thinking about your current service, what do you think needs to be changed and why?').

\section{Reflexivity}

Interviews were conducted by LN and HW who had Masters degrees and were female Research Assistants from the University of Manchester's Centre for Women's Mental Health. Both researchers were interested in mental health and working with service users in the area. Both researchers had been trained by an experienced member of the research team (TW) to conduct qualitative interviews with service users and to use qualitative research methods. The researchers had no prior relationship with the participants before the interviews were conducted.

\section{Analysis}

Interviews were audiotaped, with the participant's consent. If a service user did not consent to the interview being recorded the researcher conducting the interview made written notes throughout its duration. All interviews were transcribed verbatim and were anonymised, to protect the identity of research participants, and individually checked for accuracy by a third member of the research team. Analysis was completed by three members of the research team (LM, HW and TW), using the systematic method of thematic analysis proposed by Braun \& Clarke (2006). Data were analysed in an inductive 'bottom-up' manner, so the process was data-driven rather than theoretically driven. An essentialist 
approach (Braun \& Clarke, 2006) was applied. Each transcript was analysed by hand, by looking for patterns in the data, and noting themes or analytical categories. Themes were identified at the semantic level, focusing on what participants explicitly said, rather than any underlying latent meanings. This process continued until no new themes were found. Themes were then clustered together, noting overlaps and goodness of fit, to form categories, which are reported in the results section of this paper.

Because of time constraints, the interview transcripts were not returned to participants for comment and correction. However, participants were told that, if they requested to see the interview transcript, it would be provided. A copy of the final evaluation report was sent to services for them to disseminate the findings to service users as they wished.

\section{Ethics}

Ethical approval was granted by County Durham \& Tess Valley NHS Research Ethics Committee (10/H0905/13, $1^{\text {st }}$ Jun 2010). All services included provided, site-specific approval via NHS Research and Development Offices.

\section{Results}

Analysis of the interviews produced four major themes across the sites, with some subthemes: i) experiences of current placement versus previous placements (subthemes: having limited/no choice and experience of transition to a new unit), ii) relationships with staff, (subthemes: being treated as an individual and the importance of regular staff) iii) challenges of living with other women (subtheme: supporting each other) and, iv) having 
a voice - being involved in care and treatment. Names that have been used for quotes in this section are made up, maintaining participant confidentiality.

\section{Experiences of current placement versus previous placements}

The women reflected on their preferences and dislikes of their previous placements. These included high secure services, other medium secure services and prison. Women from WEMSS highlighted negative factors about the settings from which they had been referred. Some described those facilities as being overcrowded and commented on the lack of activities and structure to their days in comparison to WEMSS. Several women also felt that they had limited or no choice over their lives in their previous units:

'Sometimes in the last place we'd have like seventeen, eighteen on a ward. Before they cut them down for us to come here sometimes there'd be like twenty people on a ward, when I first went there, there was twenty of us on the ward. And that was weird, we were like strangers. Some people [staff] you didn't see...hardly ever...' (Brenda)

'I was on a wing with 64 women, I couldn't cope with it. You do get some nice prison officers in there who try to be understanding, but you know, for the most part, if you're ill it's prison and certainly where I was, there was no hospital wing'. (Fiona)

In common with WEMSS women, several non-WEMSS women felt that, compared with 
their current unit, previous placements had not offered adequate support and were overreliant on medication versus therapeutic engagement:

'In my old hospital we got medication, no activities - hardly any activities. Nurses wouldn't really talk to you, you know they'd dish out pills and that'. (Deborah)

'At [previous hospital name] they just used to like leave me, and all my other hospitals they just used to leave me. Even though I was there for nine years, they didn't even know me. Once, one of my other therapists at [hospital name 4], she answered her phone half way through our session'. (Penny)

When discussing their first impressions and settling in, the majority of women in WEMSS felt that they had been given adequate information about what to expect on arrival. Nevertheless, some still found the transition an overwhelming experience and reported that it took several months for them to settle in - before they could 'feel comfortable'. This tended to be in relation to women who had been transferred from prison. In contrast, women who were on the pathway through secure mental health provision reported settling in quickly and this was facilitated by unexpected levels of 'freedom' and trust placed in them by the staff:

'It was different, you have more freedom. You have more staff, as well, looking after you'. (Imogen) 
'So it was a bit like when I was on the unit at prison, it was a bit like that except better. You didn't get locked up. It takes you a good six months before you can really say you're settled'. (Fiona)

'When I got here it was a bit of a, err, errm, surprise, because you had to go into blue gowns and strip blankets and can't have property in your room...you have to ask, when you come here, for belongings through your ward round. I hated it. I wanted to go back to prison. I prefer to be here now. I call it my home'. (Alice)

In comparison with WEMSS, non-WEMSS women reported more variable first impressions of their current units. Whilst some found units friendly and welcoming on arrival, others recalled finding it confusing and intimidating. Many reported, unlike women in WEMSS, the need to have more information about units before being transferred. They often felt confused and frustrated after their arrival due to this lack of prior information coupled with unfamiliarity with the environment, its routines and procedures:

'I didn't know I was going to medium secure until I got here. No one told me it was medium secure. I would have liked to have visited here first to see what it was like before being admitted'. (Olivia)

'Confusion. Frustration, because I kept thinking that I was still within that prison environment, so I was expecting them to lock the door, my bedroom door at night and...so it was quite difficult, that stuff'. (Laura) 


\section{Relationships with staff}

All service users talked about the importance of their relationships with staff members. Women in WEMSS were generally positive about their relationships with staff using descriptors such as 'encouraging', 'caring', 'helpful', 'reassuring' and 'supportive' - often spontaneously in the interviews comparing WEMSS favorably with previous placements:

'They're quite supportive. If you've got a problem, or you want to make a complaint, the staff are really nice about it. The relationship I've got with staff is...they're very good. If I want to talk...if I want to... if I'm down here and I want to talk, they will happily talk to me. They cared and they listened to me'. (Eliza)

'You can approach staff and they can help you with anything, any personal problems or anything like that. You can just take them to a side and say, look I need help with something and they're very caring. They're very good with keeping your dignity and respect and stuff like that. The staff are really nice here. This is the best hospital I've ever been in and it is really helpful. They're dead caring, dead nice and they reassure me'. (Kate)

Women in WEMSS reported perceiving staff as wanting them to move forward and progress, and having helped them to start 'turning their lives around' and facilitating the recovery process so that they could 'move on'. In this respect, an important factor was women feeling that staff treated them as valued individuals who were worthy of respect. 
Several reported the positive effect this had on their engagement and recovery and, recursively, their attitudes to staff. Furthermore, some women reported finding staff controlling and disciplined. However, this was generally regarded in a positive light because discipline and structure were perceived as positive factors for fostering recovery:

'I feel like, erm [pause] staff are there to control us so we've [inaudible] to respect the boundaries. Yeah, and I feel to a degree that it's very disciplined'. (Alice)

'Because if they're not [consistent] you would just be all over the place'. (Imogen)

'My staff team are really good. They're quite strict with me because I need boundaries...' (Kate)

Not surprisingly, women also highlighted the importance of having regular staff on the units. WEMSS women said this was very important to them because of the need to build trusting relationships with staff that know them and understand their needs. Some women also felt that the level of training and staff experience were important in terms of developing relationships with them and they highlighted that this was important in developing positive relationships:

'The younger staff, because you get them [inaudible] university books and then they come here and they reckon they know what's in your mind. I get on really well with most of them, especially old-school staff. But as I said, the younger ones, it 
takes me time to build up trust'. (Alice)

Non-WEMSS women were also generally positive about their current relationships with staff, reporting that they were more supportive than in previous placements. The added support meant that women felt safer and staff were better able to recognise when they needed help, such as spotting indicators of low mood:

'They know when I'm low and not low. When I'm low I just go really quiet and like curl up in a ball and that, so that's when... Yeah, or I isolate myself in fleeces'. (Penny)

In common with women in WEMSS, the non-WEMSS women stressed the importance of having regular staff who know the patients. However, this view was not shared by all, with some feeling that 'staff is staff'. Further, some women felt that there were not always enough members of staff, with potentially negative consequences for women's care and safety:

"We're always complaining about the amount of staff/patient ratio. And we're always being told 'oh we're running on the highest patient ratio in the country'. Sometimes there's not enough staff and things happen. People self-harm and there's just not enough staff to go around to deal with it sometimes. They just don't seem to listen about staffing, that's the main problem. Sometimes staff are too busy here". (Olivia) 


\section{Challenges of living with other women}

All the participants talked about the dynamics of living with other women with similar needs and described how this could both help and hinder their recovery. Women in WEMSS tended to describe WEMSS as being like 'a community'. This might be because many of them have known each other for long periods. However, the ethos and design of units also appeared to have contributed to the sense of 'homeliness' and being 'just like a family'. WEMSS women also generally regarded a 'female-only' (versus mixed-sex) environment as contributing positively to their recovery - particularly in therapy sessions where being with other women enabled them to talk more openly, because they could share similar gender-specific experiences:

'With women they can trust each other and they know how you feel. Say a session about alcohol, talking about getting back out with your family, meeting your kids and stuff, we can connect with each other because that's how we feel'. (Kate)

However, some women highlighted potential difficulties of living with other women, especially in the context of high levels of observation, differing mental health issues and the problematic interpersonal dynamics that may occur as a result of such issues:

'I think it can be difficult if you've got someone on the ward who's on two-to-one obs and, the rest of the ward, the patients on the ward sort of suffer'. (Alice) 
'Because like our ward concentrates more on MIs...not MIs, PDs, and part of them is MIs, but there's some people are both, like I'm both so... but some wards have got people on them that are not suited for that ward and they disrupt the other people. So you can have nine people that are fine and one person that's disruptive. So sometimes I think they need to look more carefully at the people they put on the wards before they do it'. (Brenda)

Non-WEMSS women also reported being generally supportive of each other; however, like WEMSS women, they also reported difficulties living with other women, highlighting how individual women's behaviour could negatively affect other women and ward dynamics:

'We do help each other, but we do have our moments though, as all women do. Just because if you see one person doing the...the other person might think; well, if they can do it, I can do it. It can upset the ward as well'. (Rosie)

\section{Having a voice - being involved in care and treatment}

Participants identified the importance of being involved in their care and having a 'voice' within the service. WEMSS women spoke positively of being involved in decision-making about care and having choices about their treatment options. This had given women a greater sense of control - both in terms of their care and behaviour. According to Fiona, this made her feel '... as though I'm being put in the driver's seat'. Women reported that this engendered a sense of hope for the future. However, some of the women in WEMSS indicated that certain aspects of their life were still controlled, such as access to clinicians 
and medication management. This was not universal. Other women felt very involved in their care and reported having access to a range of treatment options and involvement in decisions about the care and support they received:

'I think they help you with choices in what activities you might do or stuff like that, what community leave you might do'. (Heather)

'Here they like to try to include you in decisions. If they want to change your medication they'll ask you for your opinion, they'll give you options and they'll discuss with you, they'll send a pharmacist to discuss it with you and like they keep you included in everything and just give you choices really and give you a bit of responsibility into your own care rather than just dictating to you'. (Clare)

'Yeah, we got asked about what we'd like here, what we think would be good for us, a lot of things we got asked about...They usually give you the therapy they think would suit you best. But then you've always...you can always say whether you think it suits you or not or if there's something you'd rather do'. (Brendan)

Accordingly, even if they disagreed with the outcome, WEMSS women reported feeling positive about their ability to contribute to the process. Support mechanisms such as advocacy services and service user forums were felt to be helpful and important resources for allowing the women in WEMSS to voice any concerns. In this regard, women reported accessing patient advocacy services such as Women in Secure Hospitals (WISH) and 
Patient Advice and Liaison Service (PALS) and finding them beneficial. Service user forums were also reported to be important as they allowed women to discuss issues and to meaningfully contribute to key processes, such as staff recruitment. Some women reported being incentivised to attend because if issues were not resolved within the forums, they could be escalated to the Trust-wide user forum and brought to the attention of senior management:

'I go to the user forum, with the directorate. I get across the ward's views and I chair it'. (Deborah)

'We have things like meetings like the user forum and stuff, that's just for women, that's a women's user forum, that's once a month I go to that, where we air our views and all our problems on the ward, not personal problems just things that affect the ward and then we bring them up and we talk to management and sort things out and get things done'. (Brenda)

Non-WEMSS participants reported that although they were given some control over their care, they would like to have been given more. The need to work together with staff was strongly voiced by the women; they felt it essential for staff to listen to them and involve them in decisions regarding their care:

'They control everything we do, really, don't they? Give us more freedom. Let us be a bit more independent, like'. (Susie) 
Women spoke about other mechanisms by which they felt involved in their own care and management of the unit, such as involvement in care planning, risk assessment, patient forums and making decisions about social activities. Participants from these sites also agreed that advocates and support services such as patient forums were a beneficial addition for them:

'They're always on the ward [advocates]. The service user will come round asking us if we've got anything we'd like to raise, as a service user, so yes, it's good. They would tell you what was going on and why, and they'd explain to you why. So it was good'. (Naomi)

\section{Discussion}

This qualitative study, embedded in the WEMSS evaluation, suggests that women in WEMSS and comparable women in MSS have very similar perspectives on what works well in their current services and what is important to them. In general, women service users expressed preferences for, and more positive first impressions of, their current versus previous placements. Women particularly liked having a range of activities and structure to their days, expressing dissatisfaction with previous services that had focused on medication rather than therapeutic activities. Women also valued being able to develop positive, trusting relationships with staff and highlighted how this could be jeopardised by the absence of regular staff. Involvement in their care and treatment were felt to be important for empowering women. The majority of women felt that their current placement 
facilitated this and gave examples such as access to advocacy services. The main differences between WEMSS and non-WEMSS women's accounts were in relation to the amount of pre-transfer information they had received and levels of staff support, with some non-WEMSS women indicating that they would like more information about services prior to admission and a higher staff/service user ratio.

Overall, these findings suggest that service users generally preferred their current placements and that WEMSS were, for the most part, meeting their aims of enhancing women's recovery through increased relational security and ability to engage in advocacy and decision-making. Similar experiences were also reported by non-WEMSS women, which might suggest that these features of an 'enhanced' service were already in existence in standard medium secure care. However, whilst non-WEMSS women spoke positively of their relationships with staff, they also described barriers to relational security such as use of agency staff, which can lead to a more custodial and less therapeutic ward environment for service users (James, Fineberg, Shah \& Priest, 1990). Low staff levels and use of bank staff have been identified as barriers to relational security in an earlier evaluation of medium secure care for women (Parry-Crooke \& Stafford, 2009). This may also contribute to staff burnout and emotional exhaustion, which research has shown to be higher in staff working on women's wards compared to those working on men's wards (Nathan, Brown, Redhead, Holt \& Hill, 2007).

It is not surprising that women from both types of services had similar experiences of relationships with staff since the concept of relational security was already present in some 
medium secure service models of care (Parry-Crooke \& Stafford, 2009). It is also possible that during the evaluation period some cross-contamination occurred between WEMSS and MSS, perhaps due to staff movement between services and transfer of information about what was working well in WEMSS.

Relationships were also discussed in the context of living with other women, again highlighting the importance of positive relationships for women in secure services. Women-only wards can bring with them a new set of challenges. Movement towards single-sex psychiatric services was driven by concerns about the safety and vulnerability of women. However, reports from women service users in medium secure care suggest that gender segregation does not necessarily enhance feelings of safety and that vulnerability to non-physical abuse such as bullying and intimidation may be increased in women-only services (Mezey, Hassell \& Bartlet, 2005). Effective management of service user relationships is an important contributor to relational security, particularly for women who have a history of complex trauma. As one service user commented, an incident can have a big impact on the safety and recovery of other women, and it is essential that interactions between patients are constantly monitored, and that women are encouraged to talk about the effect that ward dynamics have on them and their recovery (Edge, 2005). Earlier evaluations of women's MSS have identified the need for staff interventions which support peer relationships and reduce the risks of women being distressed or re-traumatised by the behaviours of other women on the ward (Parry-Crooke \& Stafford, 2009).

Another recommendation integral to gender-sensitive mental healthcare, and to the 
WEMSS model, was the principle of empowerment and giving women a voice. This approach has been emphasised throughout guidelines for gender-sensitive care strategies (Department of Health strategies, 2002; 2003; 2004) and acknowledges that a history of dysfunctional relationships and gender inequality in society may have contributed to a sense of dependence and powerlessness. Our findings suggest that both types of services were doing well in terms of involving women in their own care and enabling them to feel more in control of their lives. However, some women felt that they would like more control. Of course, this might arise from the contradiction between being detained in a secure unit against your own will and achieving a full sense of empowerment and control.

In addition to providing an enhanced needs-led therapeutic environment for women, the WEMSS pilots also aspired to facilitate women's pathways into and out of services (Sahota et al., 2010). A key stakeholder expectation was that length of stay in WEMSS would be decreased in comparison to standard medium secure care, as a result of additional resources and staff expertise. Whilst transition to lower levels of security is an important milestone in the recovery journey, women's views highlight the potential dangers of movement between services and the importance of a well-planned approach during this time. Readmission after release from a medium secure unit is common and women are 3.7 times more likely than men to be readmitted, highlighting the importance of carefully planned discharge or transitioning between services (Clarke et al., 2013). During these high risk transition periods opportunities to meet with the new team are important, as well as giving women the opportunity to work through feelings of rejection and abandonment by their previous carers (Sarkar \& di Lustro, 2011). The issue of well-planned transitioning also 
calls for transparent admission and discharge criteria, so that both women and staff can plan ahead for movement and any feelings of abandonment can be reduced.

\section{Practical implications}

This study was the first to compare views of WEMSS service users in the UK, and could have important, and achievable, implications for secure service provisions nationally. Increasing the level of meaningful activities that service users can engage in could provide for a more structured day, and a wider range of activities to get involved in. This, however, necessitates extra staff resource. A further implication relies on the need for more welltrained, and regular staff. Regular staff can allow women to develop trusting, containing relationships that have been described in this study as so vitally important for recovery.

A further implication to come from this study is the need for standardized pre-transfer information for women service users. Women in non-WEMSS services felt that they did not receive adequate information about the unit that they were being transferred to. Providing brief information leaflets or an opportunity to speak to a member of staff pretransfer is recommended, as this could lessen feelings of anxiety or abandonment. Opportunities to visit the transfer destination, or meet with members of the individual's new care team would be beneficial to ensure high quality transfer, and continuity of care.

Finally, and in keeping with WEMSS principles, women should continue to feel empowered through integral involvement in their own care. Allowing service users to attend multi-disciplinary team meetings from the beginning (as opposed to being invited in 
only at the end), to be involved in medication reviews and to be involved in their own care planning and risk assessments are relatively easy ways to increase feelings of empowerment. This way of working may have an added benefit of building trust and maintaining integrity, if staff are seen to be transparent in their decision-making processes with service users. Engagement with service user forums, Patient Advice and Liaison Service (PALS) and Women in Secure Hospitals (WISH) may allow women service users the additional chance of having their voice heard at a service-wide level, and the feeling of enabling change in a more systemic way.

\section{Strengths and limitations of this study}

This is the first study to examine and compare views of WEMSS service users and MSS service users in the UK. Purposive sampling ensures that a wide range of views is represented. In assessing the quality of the data collected in this study a number of factors were considered. First, three members of the research team independently analysed the data, reducing the likelihood of researcher bias in data interpretation and increasing interrater reliability of results. Credibility or confidence in the data was gained by the first author's prolonged engagement with the data (Lincoln \& Guba, 1985). Consistency was maintained by keeping an audit trail and this involved asking a colleague not involved in the study to check over the author's decision and analysis processes. Transferability (neutrality) was evaluated by providing the raw data to a colleague so they were able to interpret how themes had emerged. When interpreting our results, some account must be taken of the fact that this study is based on a small sample size, and the results must therefore be interpreted with appropriate caution. Further, one of the reasons as to why 
there may appear to be little difference between the views of WEMSS and MSS women may be related to the fact that many appeared to be comparing their experiences to previous poor placements (i.e. prison); this may have diluted any differences between the two models. However, the purpose of qualitative research is not to generalise from the results, but to transform and apply them to similar situations in other contexts (Polit \& Beck, 2014). It is important to note that that the findings in this study are just one of many possible interpretations, and therefore findings in this study cannot be generalised, but should be viewed as one voice in a continuing discourse.

\section{Conclusion}

The similar picture of WEMSS and MSS which emerges from the accounts of service users brings into question whether the WEMSS pilots are a unique service or whether the 'WEMSS' model of care is already being delivered in standard medium secure services. Although service users gave predominantly positive feedback about their current services, some recommendations ought to be implemented to benefit both service users and staff. Any barriers to relational security (such as staff shortages or lack of regular staff) should be addressed to ensure that women are given the opportunity to develop positive therapeutic relationships and experience continuity of care. Appropriate levels of staff will also have a positive impact on ward dynamics and peer relationships. In addition, services must ensure that women are given the appropriate support during a period of transition, either through provision of detailed information or the opportunity to meet with new care teams and settle in gradually. The accounts of women described in the current study suggest that initiatives such as Women in Secure Hospitals (WISH), Patient Advice and Liaison 
Service (PALS) and service user forums are well received by women. We would therefore recommend that initiatives such as these are included in all services and that women are given the opportunity to, and encouraged and supported to, make use of them.

\section{Availability of data and material}

This can be shared.

\section{Declarations}

Ethical approval for the study was obtained from County Durham \& Tess Valley NHS Research Ethics Committee (10/H0905/13, $1^{\text {st } J u n ~ 2010) . ~}$

\section{Acknowledgements}

The authors would like to thank all service users who were interviewed as part of this research. We would like to thank Kathryn Harney and Annie Bartlett for their expert guidance and advice. We would also like to express our gratitude to Boo Wheatcroft and the Mental Health Research Network (MHRN) for their hard work and valuable contribution, and all staff who facilitated data collection at the participating sites. 


\section{References}

Braun, V. \& Clarke, V. (2006). Using thematic analysis in psychology. Qualitative Research in Psychology, 3, 77-101. Doi: 10.1191/1478088706qp063oa

Clarke, M., Duggan, C., Hollin, C. R., Huband, N., McCarthy, L., \& Davies, S. (2013). Readmission after discharge from a Medium Secure Unit. The Psychiatrist, 37, 124129. Doi: 10.1192/pb.bp.112.039289

Department of Health. (2000). Safety, privacy and dignity in mental health units: guidance on mixed sex accommodation for mental health services. London: Stationary Office.

Department of Health. (2002). Women's Mental Health: Into the Mainstream - Strategic Development of Mental Health Care for Women. London: Stationary Office. 
Department of Health. (2003). Mainstreaming Gender and Women's Mental Health: Implementation Guidance. London: Stationary Office.

Department of Health. (2004). Women's mental health strategy. London: Stationary Office.

Edge, D. (2005). Women's Enhanced Medium Secure Services (WEMSS): A scoping exercise for developing a research and evaluation strategy. London: Stationary Office \& National Offender Management Service.

Edge, D., Walker, T., Meacock, R., Wilson, H., McNair, L., Shaw, J., Gutridge, K., Mitchell, H., Robinson, L., Senior, J., Sutton. M. \& Abel, K. (2017). Secure Pathways for Women in the UK: Lessons from the Women's Enhanced Medium Secure Services (WEMSS) Pilots. Journal of Forensic Psychiatry and Psychology, 28 (2,) 206-225 http://dx.doi.org/10.1080/14789949.2016.1244279

Grubich, C. (1999). Qualitative research in health. An introduction. Quebec, Canada: Allen and Unwin.

Howlett, M. (1994). Special Hospitals Service Authority Service for Women Patients. London: SHSA. 
James D. V., Fineberg N. A., Shah A. K., \& Priest R. G. (1990). An increase in violence on an acute psychiatric ward: a study of associated factors. British Journal of Psychiatry, 156, 846-852. Doi: 10.1192/bjp.156.6.846

Kidder, L. H. (1981). Qualitative research and quasi-experimental frameworks. In Brewer M.B., Collins B.E., (Eds.), Scientific Inquiry and the Social Sciences (pp. San Francisco, CA: Jossey-Bass.

Lincoln, Y. S. \& Guba, E. (1985). Naturalistic Inquiry. Thousand Oaks, CA: Sage.

Mays, N. \& Pope, C. (1995). Qualitative research: Rigor and qualitative research. BMJ, 311, 109-112. Doi: 10.1136/bmj.311.6997.109

Mezey, G., Hassell, Y., \& Bartlett, A. (2005). Safety of women in mixed-sex and singlesex medium secure units: Staff and patient perceptions. British Journal of Psychiatry, 187, 579-582. Doi: 10.1192/bjp.187.6.579

Miller, W. L., \& Crabtree, B. F. (1992). Primary care research: A multimethod typology and qualitative road map. In Crabtree, B.F., \& Miller, W., L. (Eds.), Doing qualitative research. Research methods for primary care (Vol. 3). Newbury Park, CA: Sage.

Nathan, R., Brown, A., Redhead, K., Holt, G., \& Hill, J. (2007). Staff responses to the 
therapeutic environment: A prospective study comparing burnout among nurses working on male and female wards in a medium secure unit. Journal of Forensic Psychiatry and Psychology, 18, 342-352. Doi: 10.1080/14789940701441136

Patton, M., Q. (1990). Qualitative Evaluation and Research Methods. Newbury Park: Sage. Parry-Crooke, G. \& Stafford, P. (2009). My life: in safe hands? Dedicated women's medium secure services in England. London: London Metropolitan University.

Polit, D. \& Beck, C. (2014). Essentials Of Nursing Research: Appraising Evidence For Nursing Practice. $8^{\text {th }}$ ed. Philadelphia: Lippincott Williams \& Wilkins Health.

Reed, J. (1992). Review of Health and Social Services for mentally disordered offenders and others requiring similar services: Final Summary Report. London: Stationary Office.

Royal College of Psychiatrists. (2000). Your guide to relational security: See think Act. $2^{\text {nd }}$ edition. London: Royal College of Psychiatry.

Sahota S., Davies S., Duggan C., Clarke M., Huband N., \& Owen V. (2010). Women admitted to medium secure care: their admission characteristics and outcome as compared to men. International Journal of Forensic Mental Health, 9, 110-117. Doi: $10.1080 / 14999013.2010 .499555$ 
Sarkar, J. \& di Lustro M. (2011). Evolution of secure services for women in England. Advances in Psychiatric Treatment, 17, 323-31. Doi: 10.1192/apt.bp.109.007773

Shaw, J., Davies, J. \& Morey, H. (2001). An assessment of the security, dependency and treatment needs of all patients in secure services in a UK health region. Journal of Forensic Psychiatry, 12, 610-638. Doi: 10.1080/09585180127380

Walker, T. \& Doyle, M. (2009). A qualitative service evaluation of service user's and health care professional's experiences of the therapeutically enhanced medium-secure service for women (TEMSS $(W))$, a report produced for Greater Manchester West Mental Health NHS Foundation Trust.

Women In Secure Hospitals (1999). Defining Gender Issues: Redefining Women's Services. WISH. 